\title{
Analysis of Data on the Relation between Eddies and Streaky Structures in Turbulent Flows Using the Placebo Method
}

\author{
S. I. Chernyshenko, G. M. Di Cicca, A. Iollo, A. V. Smirnov, \\ N. D. Sandham, and Z. W. Hu
}

Received January 25, 2006

\begin{abstract}
An artificially synthesized velocity field with known properties is used as a test data set in analyzing and interpreting the turbulent flow velocity fields. The objective nature of this approach is utilized for studying the relation between streaky and eddy structures. An analysis shows that this relation may be less significant than is customarily supposed.
\end{abstract}

Keywords: turbulence, visualization, streaky structures, eddy, Galilean decomposition.

Until recently it was generally accepted that the existence of so-called streaky structures in turbulent wall flows was dictated by the presence of eddy structures [1]. Now it has been shown [2] that in reality the streaky structures develop regardless of the eddy structures, since the approach which involves rejecting the assumption of a relation between the eddy and streaky structures gives extensive qualitative and quantitative results that are consistent with the experiments. At the same time, over several decades in both physical and numerical experiments the data usually interpreted as proof of the relation between streaky and eddy structures were collected. In the light of the results of [2] the existence of these data can be explained in two ways. We can assume that the streaky structures, which do not themselves depend on the eddy structures, cause the eddies thus leaving on them the imprint of their structure. The second possibility is that the generally accepted interpretation of the data is erroneous. In fact, modern eddy identification techniques were developed under conditions in which the presence of a relation between the streaky structures and eddies was assumed to be so obvious that identification methods which did not confirm this relation could be rejected as necessarily ineffective.

The present study represents an attempt to determine which of these two possibilities is the more likely. For this purpose a placebo is constructed, that is a velocity field resembling a turbulent one with streaky structures and eddies but such that the streaky structures and the rest of the field are definitely not interrelated. Then the placebo and samples of real turbulent velocity fields are processed using the same methods and the results are compared.

\section{FORMULATION OF THE PROBLEM}

Modern experimental and numerical methods give large data arrays in the form of two- and threedimensional velocity fields. The analysis and interpretation of these fields represent a complex problem for solving which various methods have been developed. It is difficult to interpret the vector velocity fields directly. In many cases the data are processed in some way, then visualized, and after that the researcher interprets the result of the visualization. For example, Galilean decomposition can be used to detect eddies. This method reduces to subtracting a constant vector from the vector velocity field. This is equivalent to transition to a traveling reference system. Then the graphic representation of the velocity field obtained is visually analyzed. In this pattern an observer can frequently see certain structures, for example, eddies. Statistical methods can be used to confirm this observation. Another effective eddy detection method consists 
in calculating the so-called "swirling strength" and visualizing the zones in which it is high. These and many other methods are described, for example, in [3] (see also [4]).

The presence of processing and visualization stages in the data interpretation process can lead to certain features of the pattern obtained being inherent in the data processing and visualization processes themselves, but not in the initial data. In the present study it is shown that this phenomenon actually takes place and a method of overcoming it is proposed.

\section{PLACEBO METHOD}

A placebo is used to verify the interpretation of the experimental data objectively under conditions in which the experiment or its interpretation are subject to the action of unknown factors. For example, in medicine in testing a new drug some patients are given a placebo (i.e. a neutral inert substance). Then the results of treatment with the new drug and the placebo are processed using the same method and compared.

As regards the interpretation of turbulent velocity fields, the placebo or set of test velocity fields may consist of artificially synthesized vector fields whose structure can be explicitly controlled. Such kinematic turbulence models were designed and used for various purposes in [5-7].

In particular, in [5] the velocity field was constructed in the form of a superposition of the fields induced in one case by randomly distributed horseshoe vortices and in another by packets of such vortices and it was shown that in the second case the agreement with the experimental data is better. Thus, in this study two different interpretations of the same experimental data were compared. This is similar but not identical to the technique for testing the validity of a single interpretation discussed below.

In the present study the placebo idea is applied to observations of the relation between streaky structures and eddies in turbulent wall flows. The experimental and numerical data samples consist of sets of velocity fields in planes parallel to the wall. The placebo samples are generated as the sum of a constant average velocity, a streaky velocity distribution which has only a single component that varies in accordance with a sinusoidal law as a function of the transverse coordinate, and a random homogeneous isotropic vector field. More specifically,

$$
\begin{aligned}
& u=14.06-2.889 \cos \left(0.02 \pi z+s_{\text {rnd }}\right)+u_{\text {rnd }}(x, z) \\
& v=v_{\text {rnd }}(x, z) \\
& w=w_{\text {rnd }}(x, z)
\end{aligned}
$$

Here, $u, v$, and $w$ are the streamwise, normal to the wall, and spanwise velocity components, respectively, $x$ and $z$ are the streamwise and spanwise coordinates, $s_{\text {rnd }}$ is a random quantity uniformly distributed over $[0,2 \pi]$, and the vector field $\left(u_{\mathrm{rnd}}(x, z), v_{\mathrm{rnd}}(x, z), w_{\mathrm{rnd}}(x, z)\right)$ is random, homogeneous, and isotropic. All the quantities in this paper are expressed in wall units. For the length a single wall unit is equal to $v \sqrt{\rho / \tau}$ and for the velocity to $\sqrt{\tau / \rho}$, where $v$ is the kinematic viscosity, $\rho$ is the density, and $\tau$ is the time-average tangential stress on the wall. The constant and sinusoidal parts correspond to the velocity profile used in [8] in analyzing the stability of a streaky structure and calculated in the plane $y=36$ wall units for the Reynolds number calculated from the dynamic velocity $\operatorname{Re}_{\tau}=360$. In this case the particular values of the coefficients indicated in (2.1) were obtained, but from the standpoint of the present study any other sinusoidal velocity profile with appropriate characteristics would give analogous results. The random vector fields were constructed using the method of [6]. These solenoidal fields are homogeneous and isotropic, have a characteristic length scale equal to 7.2 and $\left\langle u_{\text {rnd }}^{2}\right\rangle=\left\langle v_{\text {rnd }}^{2}\right\rangle=\left\langle w_{\text {rnd }}^{2}\right\rangle=0.25$. The velocity fields obtained are three-dimensional but in what follows we will use only two velocity components in the plane parallel to the wall. For this reason only $x$ and $z$ are explicitly shown as arguments of the random field in (2.1).

The sum of the average and streaky placebo components has only a single velocity component, i.e., represents a purely shear flow without eddies. However, it contains streaky structures, i.e., elongated zones in which the streamwise velocity component is less than its average value. As distinct from real streaky structures, in the placebo these structures are strictly rectilinear and infinitely long. This is sufficient for the purposes of our investigation. Naturally, the random velocity field $\left(u_{\text {rnd }}, v_{\text {rnd }}, w_{\text {rnd }}\right)$ contains eddies. 


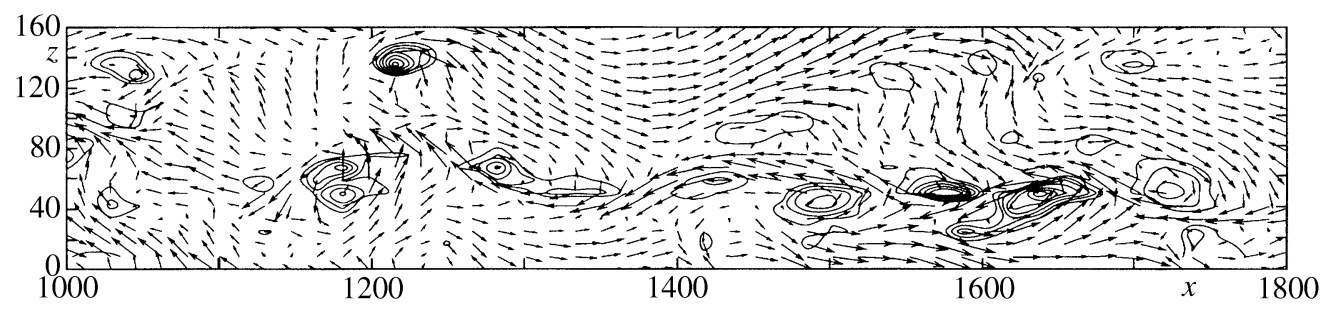

Fig. 1. Streaky structure and eddies obtained as a result of direct numerical simulation and visualized using Galilean decomposition with $U_{c}=11$ and isolines of $\Delta$; flow from left to right

From the construction of the placebo field these eddies are completely independent of the streaky structures. Consequently, although in the placebo these structures are not too realistic, this placebo can serve as a simple test group for those methods of data interpretation which aim to establish the existence of a relation between streaky and eddy structures.

\section{GALILEAN DECOMPOSITION AND ANALYSIS OF THE SWIRLING STRENGTH}

In Fig. 1 we have reproduced a streaky structure and eddies obtained as a result of the direct numerical simulation of a plane-channel flow [9]. The Reynolds number calculated from the dynamic velocity and the channel half-width $\operatorname{Re}_{\tau}=360$. The figure plane is parallel to the wall and located at a distance $y \approx 20$ from it. The average velocity $U \approx 12.0$ in the figure plane. Galilean decomposition was used with the reference system velocity $U_{c}=11$ and then the velocity was scaled by a factor of 5 to make the vectors clearly visible in the figure. In other words, the length of the velocity vectors laid off is equal to $5\left(u(x, z)-U_{c}, w(x, z)\right)$, where $u(x, z)$ and $w(x, z)$ are the velocity components obtained in the numerical calculation. In this case, instead of the three-dimensional expression for the swirling strength we need to use its two-dimensional equivalent $\Delta$ determined from the formula

$$
\Delta=\left(\frac{\partial u}{\partial x}+\frac{\partial w}{\partial z}\right)^{2}-4\left(\frac{\partial u}{\partial x} \frac{\partial w}{\partial z}-\frac{\partial w}{\partial x} \frac{\partial u}{\partial z}\right)
$$

In Fig. 1 the curves are isolines of $\Delta$ with an initial value equal to $-2000 / 360^{2}$, step equal to $-2000 / 360^{2}$, and final value equal to $-14000 / 360^{2}$. Galilean decomposition and analysis of the swirling strength are widely used eddy identification techniques [3]. At each point in the flow zone there exists a traveling reference system such that the flow velocity in it is equal to zero at this point. Large negative values of $\Delta$ indicate the points at which in this traveling reference system the instantaneous vector lines of the velocity have a spiral structure. Consequently, the zones with large negative $\Delta$ visualize eddies traveling at the local flow velocity. Ordinary Galilean decomposition visualizes eddies traveling at a velocity close to $U_{c}$.

In Fig. 1 we can clearly see that the eddies are aligned in a horizontal row in the neighborhood of the streaky structure, whereas far away from it there are almost no eddies. This can be interpreted as an illustration of the close connection between eddies and streaky structures. A similar approach is taken, for example, in [10].

We will now apply the same techniques of Galilean decomposition and analysis of the swirling strength to the placebo field in which the streaky structures and other field components are independent by design. In Fig. 2 we have reproduced the result. In the case shown in Fig. $2 U_{c}=14$, the scale coefficient for representing the vectors is equal to 3.6 , and the $\Delta$ levels begin from $-2000 / 360^{2}$, have a step equal to $-2000 / 360^{2}$, and a maximum equal to $-50000 / 360^{2}$. In this case it turns out that the eddies also form horizontal rows aligned with the streaky structure. In the placebo this effect is not so clear because in Fig. 2 the streaky structure is thicker. Nevertheless, if we simply calculate the clearly expressed eddies intersected by the curves at $z=-30$ and 30, i.e., at the streaky structure edges, and compare their number with the number of clearly expressed eddies intersected by the curve $z=0$, i.e. located in the center of the streaky structure, the difference becomes obvious. 


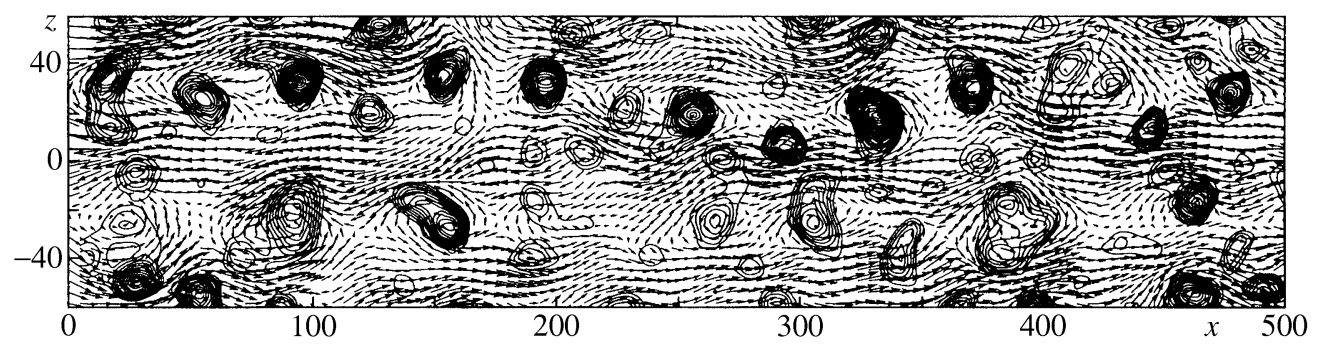

Fig. 2. Streaky structures and eddies in the placebo

The reason for the similar behavior can be seen from the formula for $\Delta$. For simplicity's sake, we will consider the case of purely two-dimensional flow in the $x, z$ plane. Then from the continuity equation

$$
\frac{\partial u}{\partial x}+\frac{\partial w}{\partial z}=0
$$

it follows that

$$
\Delta=+4\left(\frac{\partial u}{\partial x}\right)^{2}+4 \frac{\partial w}{\partial x} \frac{\partial u}{\partial z}
$$

So, if $\Delta<0$, then $(\partial w / \partial x)(\partial u / \partial z)<0$. Thus, if $\Delta<0$, an increase in $|\partial u / \partial z|$ leads to an increase in $|\Delta|$.

A similar effect also takes place in the general three-dimensional case.

In purely shear flow $\Delta=0$. However, if a shear is added to an already existing field with $\Delta<0$, then $\Delta$ changes. From Fig. 2 we can see that as a result of such change the eddies of the random component become more marked in the zones of strong shear of the streaky component of the field. Needless to say, the Galilean decomposition also shows eddies adjacent to the streaky structure since this decomposition and an analysis of the swirling strength usually give similar results.

For the purposes of the following discussion it is necessary to consider the definition of an eddy. One of the most popular definitions is as follows: the eddy is a zone in which in the plane perpendicular to the eddy axis the instantaneous streamlines have an approximately circular or spiral structure in the reference system traveling with the eddy center velocity $[11,12]$. If we adopt this definition, then in the placebo field there is a statistical dependence between the eddies and the streaky structures, that is, the eddies tend to line up along the streaky structure edges. At the same time, from the placebo design method it is known that the streaky structures have no statistical relation with the rest of the flow field. For example, we cannot say that the streaky structures were induced by the eddies around them. In other words, the detection of such a statistical relation is of no significance for the dynamics. The observed relation is only an artefact (artefactum - something artificially made, produced) created by Galilean decomposition and the analysis of the swirling strength.

Consequently, in the numerical data in Fig. 1 the observed relation between the streaky structures and the eddies may also be of no significance for the dynamics, that is, it may be only an artefact obtained as a result of using specific data processing methods. In other words, having adopted the above definition of an eddy, we can say that the eddies line up along the streaky structure edges in the experimental or numerical data but this observation cannot subsequently be regarded as evidence of the fact that, for example, the eddies generate the streaky structures (or that the streaky structures generate the eddies) in some dynamic process. A definition of an eddy which has such a property seems to be not very useful.

In order to be able to consider dynamically significant correlations between eddies and streaky structures we can introduce another definition of the eddy. Objects which satisfy the above definition will be called classical eddies. We introduce the notion of a true eddy as a classical eddy of the vector field obtained from the initial field by subtracting all other ordered structures (such as streaky structures), apart from the eddies themselves. If we could observe the presence of a statistical dependence between the true eddies and 


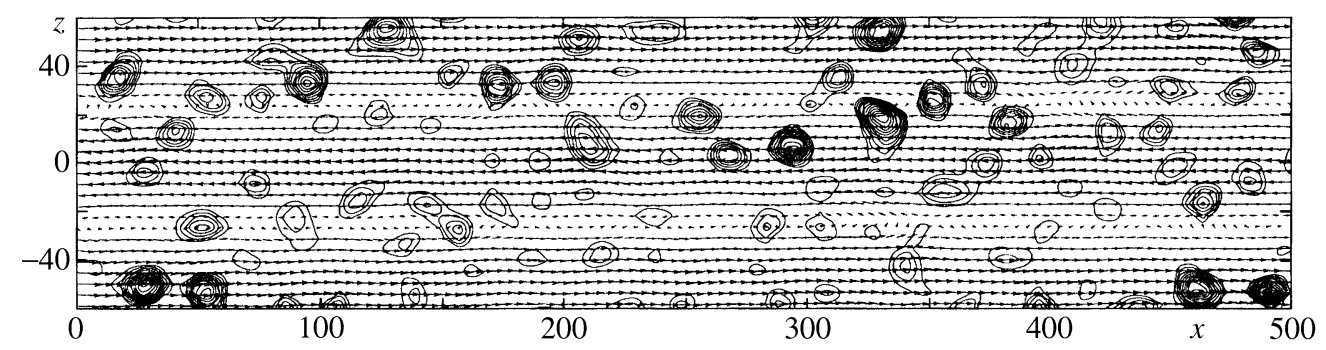

Fig. 3. Streaky structures in the reconstructed placebo field and eddies in the residual field

the streaky structures, this observation would be of significance for the dynamics. The need to subtract the streaky structures leads naturally to the idea of filtering the velocity field considered in the next section.

\section{FILTRATION}

For the placebo field introduced above the streaky structures can be the separated from the eddies using a filter based on the technique of orthogonal decomposition over a special basis which ensures maximum energy content in the minimum number of modes [13]. Thus, for a given set of velocity fields $\mathbf{u}_{k}$ we can find a vector field $\mathbf{e}_{1}$ and coefficients $A_{1 k}$ such that $\sum_{k}\left(\mathbf{u}_{k}-A_{1 k} \mathbf{e}_{1}\right)^{2}$ is minimized. Then $\mathbf{e}_{1}$ will be the first expansion mode. We can now represent each field $\mathbf{u}_{k}$ as the sum of the reconstructed and residual fields $\mathbf{u}_{k}^{\text {rec }}=A_{1 k} \mathbf{e}_{1}$ and $\mathbf{u}_{k}^{\text {res }}$. Then this procedure can be repeated for the residual fields to obtain the second mode. In practice the calculations are carried out using more effective algorithms which make it possible to obtain all the necessary modes at once. In the general case the reconstructed field can be constructed for any given number $M: \mathbf{u}_{k}^{\text {rec }}=\sum_{i=1}^{M} A_{i k} \mathbf{e}_{i}$. Then the residual fields $\mathbf{u}_{k}^{\text {res }}=\mathbf{u}_{k}-\mathbf{u}_{k}^{\text {rec }}$ will have the minimum possible energy $\sum_{k}\left(\mathbf{u}_{k}^{\text {res }}\right)^{2}$ for a given $M$.

The elements $\mathbf{u}_{k}$ of the data sets used in filtering are called windows. Each window represents a velocity distribution on a grid in a rectangular domain. The placebo fields were generated in windows whose transverse dimension was a whole multiple of the transverse period of the streaky structures. As might be expected, in this case the reconstructed field with only three modes fairly closely simulates the mean flow and streaky structures, whereas the residual field almost coincides with the random component in (2.1). Naturally, the visualization of the streaky structures in the reconstructed placebo and the visualization of the true eddies in the residual field reveal their independence (compare Figs. 2 and 3). In both figures the isolines are plotted starting from the same level and with the same step.

The results of the numerical calculation of a plane-channel flow [9] were represented in the database [14] in the form of 12 realizations of a three-dimensional instantaneous velocity field on a three-dimensional grid in a $4320 \times 720 \times 2160$ domain (streamwise, normal to the wall, and spanwise dimensions, respectively). The realizations are assumed to be statistically independent. In the present study we used data cuts produced by planes parallel to the wall. In order to increase the data volume for the analysis the cuts were taken at fixed distances from both walls so that from each realization we obtained two cuts. Although in each pair of cuts the data are not statistically independent, it may be expected that at least the fine-scale structures remain independent. In order to increase the number of samples for analysis still further, each cut was divided into $1080 \times 540$ windows (streamwise and spanwise dimensions, respectively). Each window contained $64 \times 64$ grid nodes. Of course, the windows obtained from the same cut are not completely independent. Altogether we obtained 384 windows.

The filtration was carried out for various values of $M$ and the reconstructed and residual fields obtained were investigated using Galilean decomposition and visualization of the swirling strength. It turned out that when $M=60$ the streaky structures and eddies fell into the reconstructed and residual fields, respectively. When $M=60$ on average approximately $98.1 \%$ of the energy was contained in the reconstructed field. 


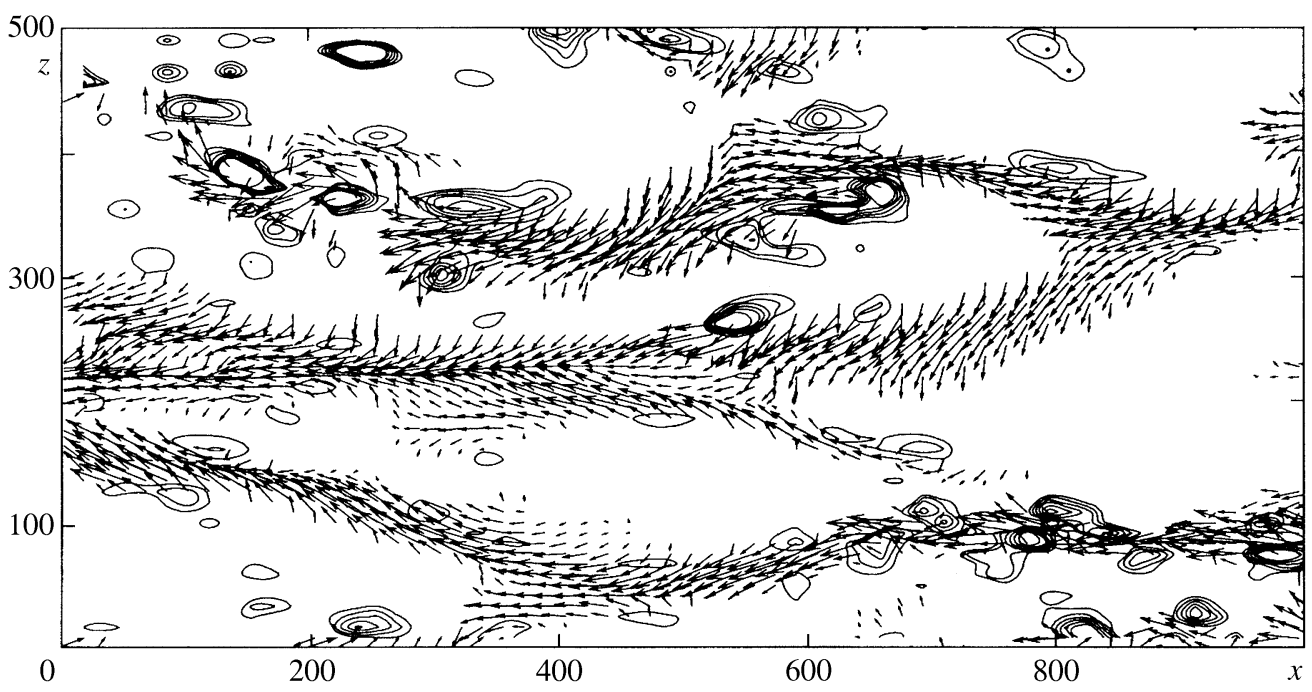

Fig. 4. Unfiltered data of a numerical calculation

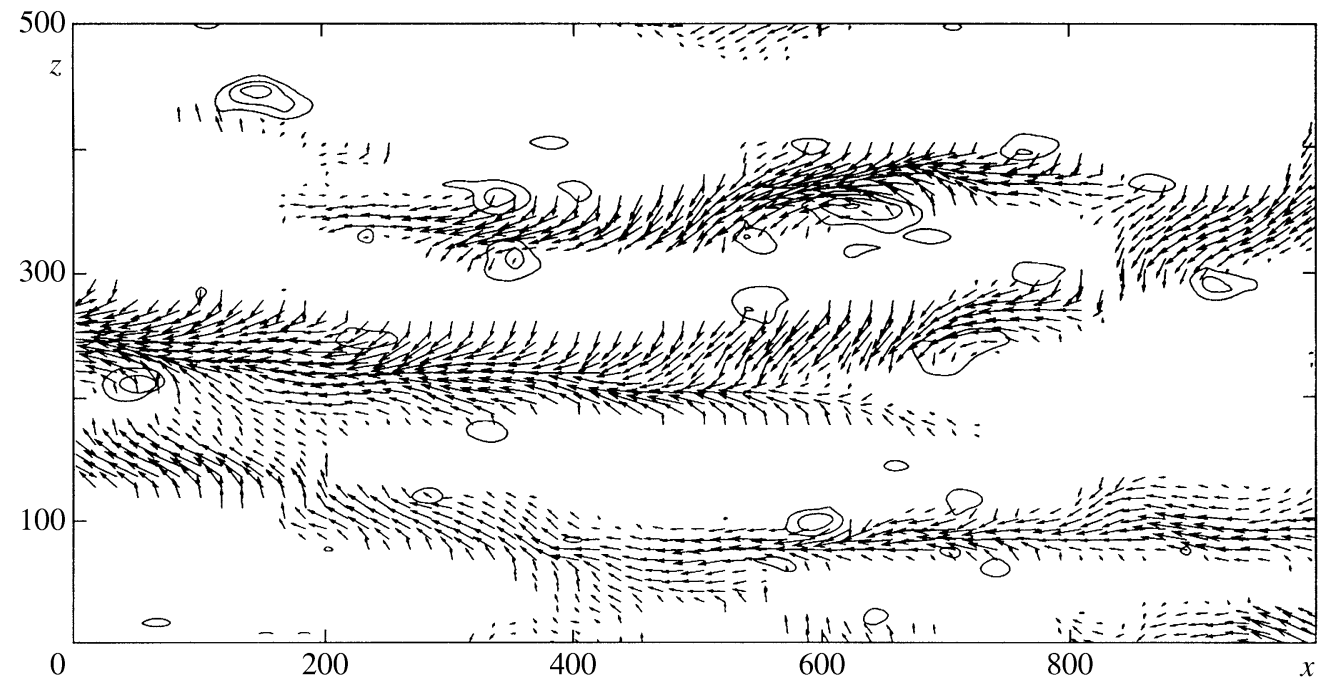

Fig. 5. Reconstructed field for the numerical calculation data

Of course, 384 windows are insufficient for all the 60 modes obtained to be correct. Consequently, the procedure described should simply be regarded as a definition of the filter used.

In Fig. 4 we have reproduced the streaky structures and eddies in the unfiltered data of a numerical calculation. The streaky structures were distinguished by including in the pattern only those velocity vectors whose streamwise component becomes negative as a result of Galilean decomposition. The eddies have been visualized by means of the swirling strength isolines. Clearly, the eddies are concentrated in the neighborhood of the streaky structures.

In Fig. 5 we have reproduced the reconstructed velocity field. The streaky structures visible in Fig. 4 are also clearly distinguishable in Fig. 5, whereas the eddies have almost disappeared.

In Fig. 6 weak traces of the streaky structures can be seen only in the lower part of the residual field. At the same time there are many strong eddies. Consequently, although not completely, the filtration has separated the streaky structures and eddies and hence the eddies in the residual field are true eddies and their visualization in the residual field by means of the swirling strength (or $\Delta$ ) are not subject to the strong influence of the streaky structures.

In Fig. 7 the streaky structures of the reconstructed field and the eddies of the residual field have been 


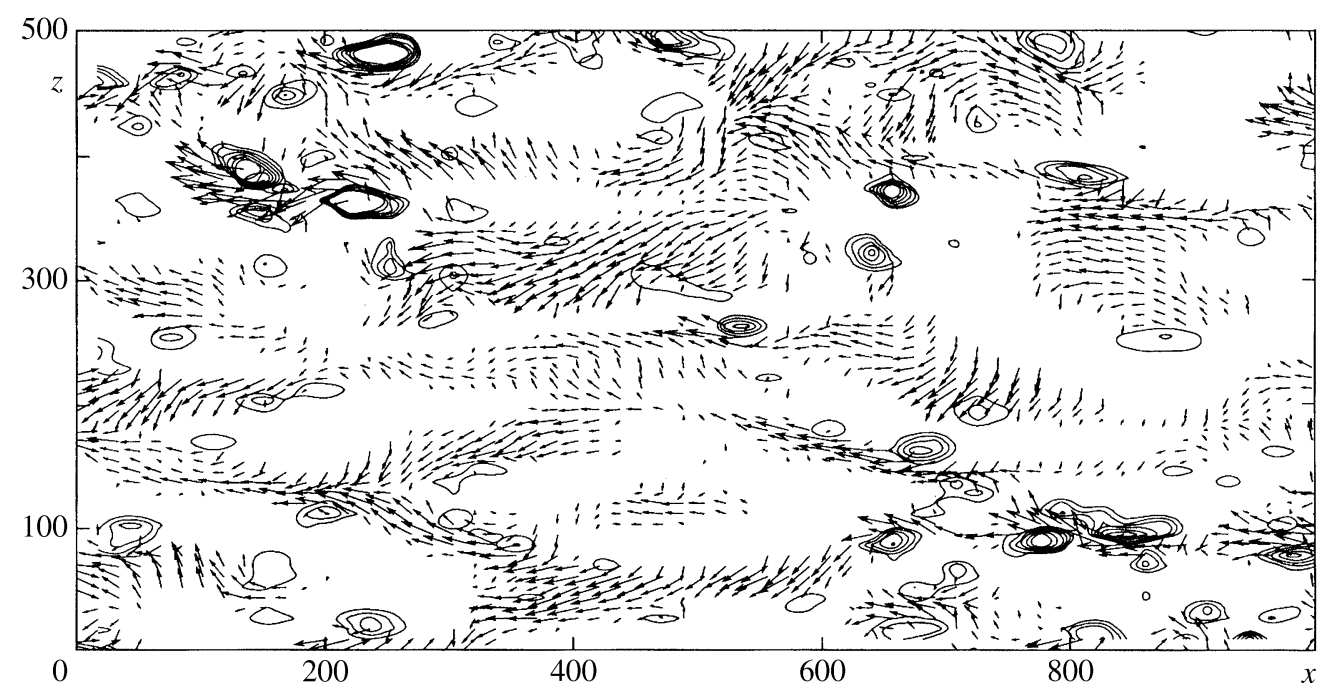

Fig. 6. Residual field for the numerical calculation data

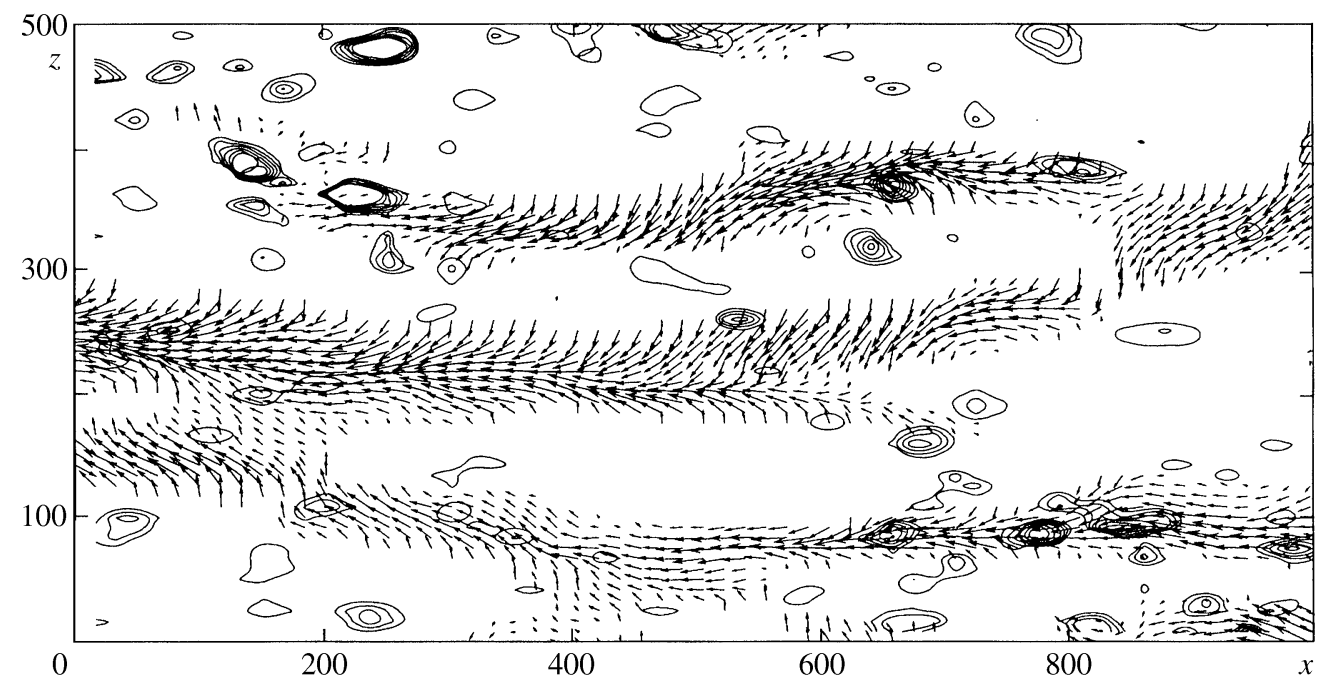

Fig. 7. Streaky structures of the reconstructed field and eddies of the residual field for the numerical calculation data

combined. Two effects are visible. Firstly, the concentration of the eddies in the neighborhood of the streaky structures is less in Fig. 7 than in Fig. 4. Secondly, although to a lesser extent, the filtered eddies continue to be concentrated in the neighborhood of the streaky structures. At least, this is the impression receiving after viewing many similar patterns. A more objective approach to estimating the relation between the streaky structures of the reconstructed field and the eddies of the residual field (i.e., between the streaky structures and the true eddies in our terminology) will be described in the next section.

A similar analysis was also carried out on a set of experimental data [15] for a boundary layer flow. These data are contained in 596 files, each consisting a single window of data including the values of two velocity components in a plane parallel to the wall in a grid of $59 \times 59$ nodes. The window size is $525 \times 525$.

Filtration of the experimental data with the same number of modes $M=60$ as for the data of the numerical calculation also made it possible to separate the streaky structures from the eddies. In this case approximately $90 \%$ of the energy was contained in the reconstructed field. As compared with the data of the numerical calculation, in the experimental data it is more difficult to observe the relation between the streaky structures and the eddies. In Fig. 8 we have presented an example of the unfiltered experimental data. 


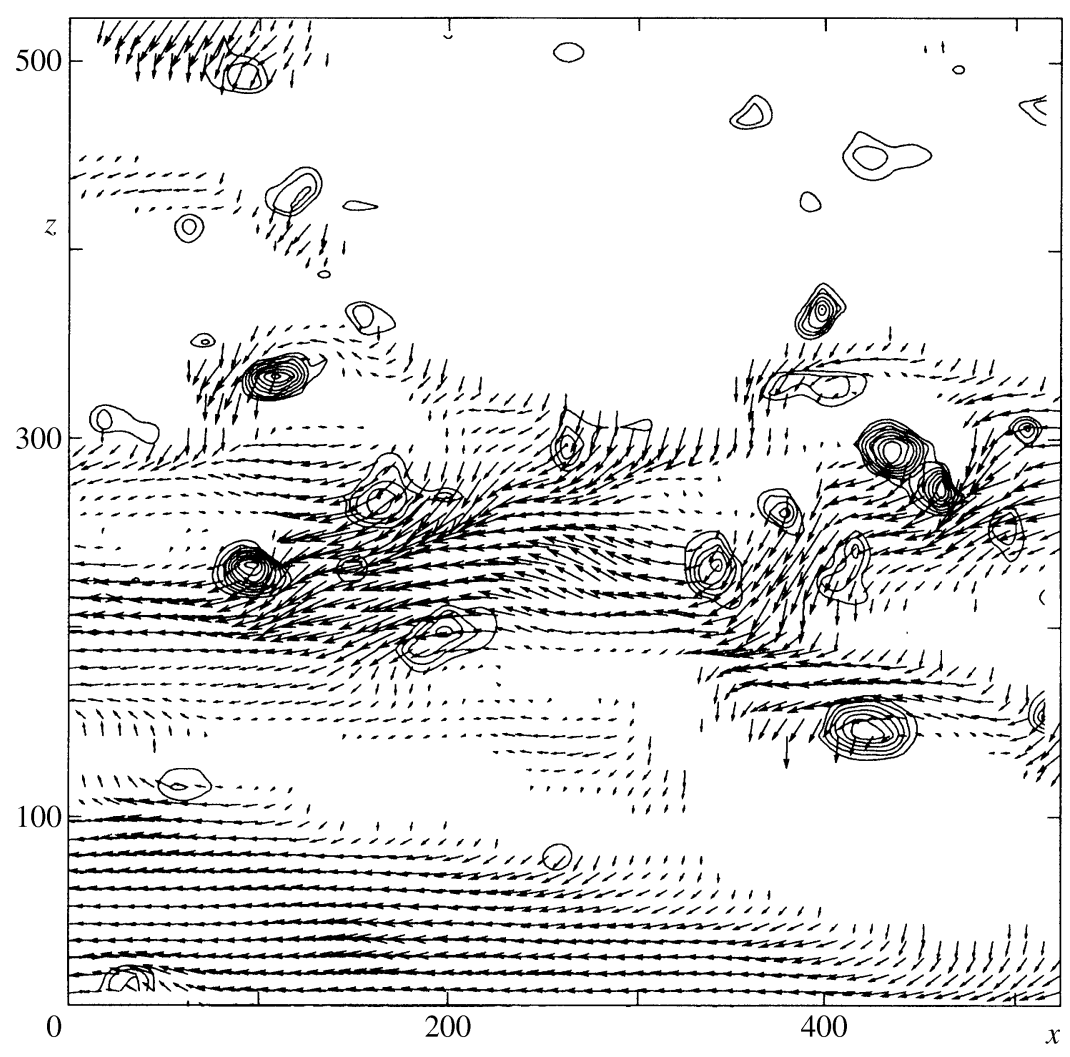

Fig. 8. Unfiltered experimental data

In Fig. 9 the streaky structures of the reconstructed field are superimposed on the eddies of the residual field for the same experimental data sample. After filtering the reconstructed field contains streaky structures but has almost no eddies. At the same time, as distinct from the case of the numerical calculation data, in the residual field the eddies are less numerous (or the eddies are weaker) than in the unfiltered field. This means that to a considerable extent the eddies observed in the unfiltered field in Fig. 8 are an artefact created by the visualization method. However, there is an impression that the true eddies which are nevertheless present in the residual field are correlated, albeit only weakly, with the streaky structures.

The above observations of the presence and degree of the relation between the streaky structures and the eddies are based on a visual impression. In the next section the probability density of the joint distribution of the streamwise velocity and the swirling strength will be used to supplement these observations with a more objective method. This approach will also demonstrate the restrictions imposed by the use of the extremely simple form of placebo employed in the present study.

\section{PROBABILITY DENSITY}

Since the streaky structures are zones of low streamwise velocities $u$ and the eddies are zones of large negative values of the two-dimensional swirling strength $\Delta$, considerable information on their interdependence is contained in the probability density $F(u, \Delta)$ of their combined distribution. The probability density is so defined that for infinitely small $d u$ and $d \Delta$ the probability of the streamwise velocity taking values between $u$ and $u+d u$ and the swirling strength values between $\Delta$ and $\Delta+d \Delta$ is equal to $F(u, \Delta) d u d \Delta$. For each window set used in the filtration we carried out a calculation of the relative frequency of incidence of $u$ and $\Delta$ in cells of the grid in the $u, \Delta$ plane. Thus, we obtained an approximate value of $F(u, \Delta)$ at the center of each cell.

When $u$ and $\Delta$ are statistically independent, the probability density can be represented as the product of functions of a single argument $F(u, \Delta)=F_{u}(u) F_{\Delta}(\Delta)$. In analyzing the probability density, statistical 


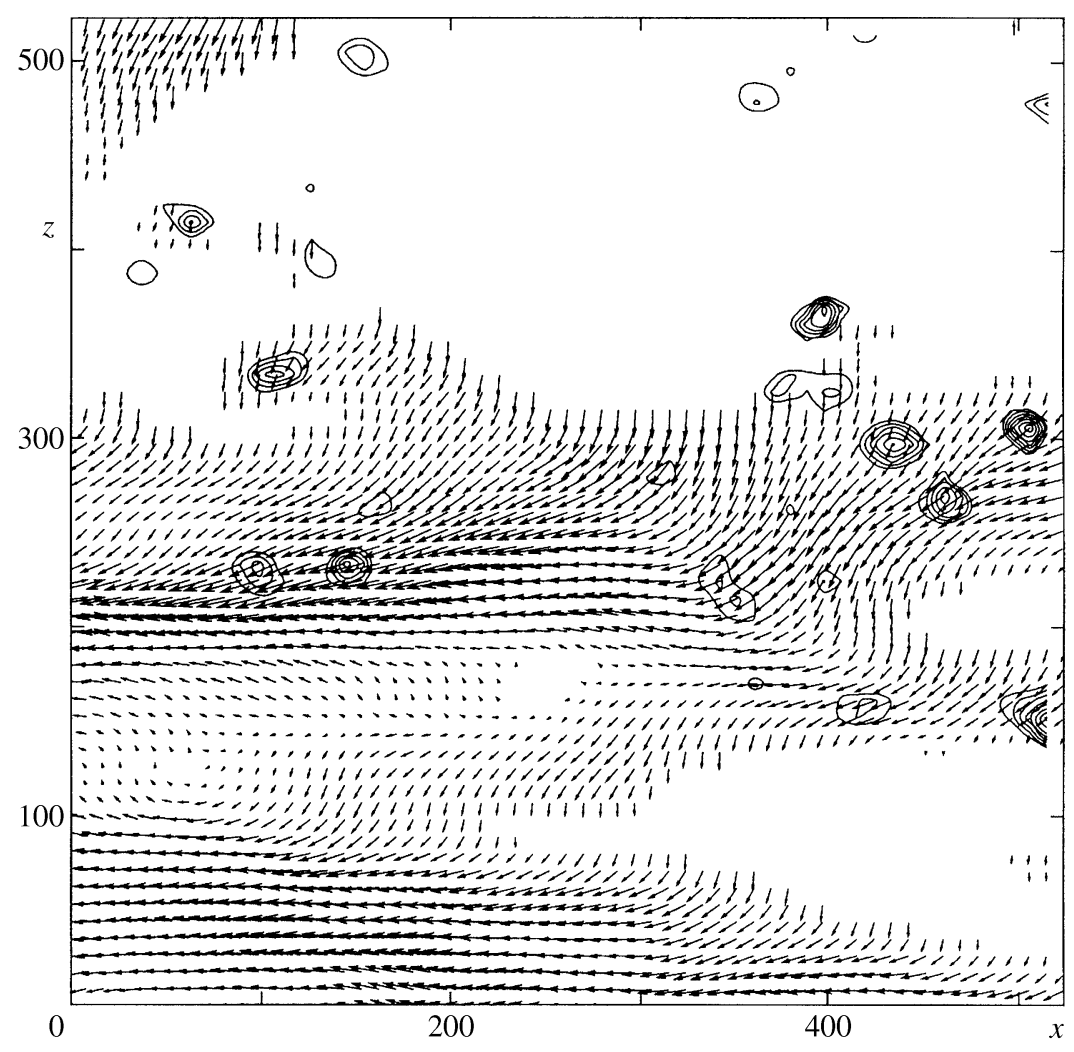

Fig. 9. Streaky structures of the reconstructed field and eddies of the residual field for the experimental data

independence can be detected on the basis of the similarity of the $F(u, \Delta)$ profiles as functions of one argument for various values of the other argument. In Fig. $10 a$ we have plotted the probability density isolines for unfiltered placebo data. The relatively large negative values of $\Delta$ correspond to eddies. Since $F(u,-0.15)$ reaches a maximum at $u \approx 14$, this means that in the placebo the eddies have a tendency to concentrate in zones with the average streamwise velocity which, of course, are also high shear velocity zones. The placebo field consists of alternating strips of high and low streamwise velocity and the eddies are concentrated in the space between these zones. At the same time, $F(u, 0)$ has two maxima at $u \approx 11.5$ and 16. Of course, this behavior is natural for a sinusoidal velocity profile and the deviation from symmetry about $u=14$ characterizes the degree of accuracy with which the probability density was obtained. The significant difference between the shapes of the profiles $F(u,-0.15)$ and $F(u, 0)$ shows that $u$ and $\Delta$ are in fact statistically dependent. This result changes sharply if the eddies and the streaky structures are previously separated by filtration. Figure $10 a$ shows that $F\left(u^{\text {rec }}, \Delta^{\text {res }}\right) \approx F\left(u^{\text {rec }}\right) F_{\Delta}\left(\Delta^{\text {res }}\right)$, as it should because of the way in which the placebo was designed.

The application of the same approach to the numerical calculation data gives a completely different pattern. In Fig. $11 a F(u, 0)$ has only a single peak at $u \approx 14$, whereas $F(u,-0.2)$ reaches a maximum at $u \approx 11.5$. This means that the eddies are more frequently observed in the reduced velocity zones, i.e., in the neighborhood of the streaky structures. It is interesting to note that the zones with large positive values of $\Delta$ also occur more frequently at smaller values of $u$, i.e., in the neighborhood of the streaky structures. As distinct from the placebo case, similar behavior can also be observed in the combined probability density of the reconstructed $u$ and residual $\Delta$, as can be seen in Fig. $11 b$. Here, the maxima of $F$ at $\Delta^{\text {res }}=0$ and -0.02 are reached at $u^{\mathrm{rec}}=12.5$ and 11 , respectively. The distance between these maxima can be regarded as a quantitative measure of the relation between the streaky structures and the eddies. For the unfiltered field this distance is equal to 2.5 , whereas in the filtered case it falls to 1.5 while remaining nonzero. This result objectively confirms the visual impression of a statistical dependence between the streaky structures and the true eddies in the numerical calculation results. 

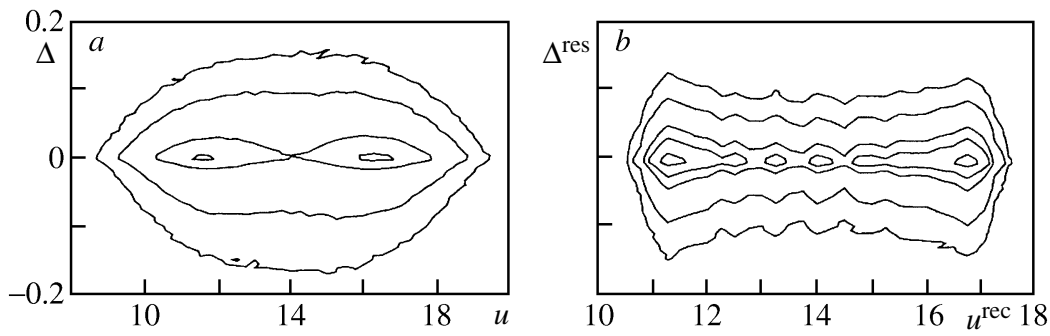

Fig. 10. Probability density isolines for the placebo: unfiltered $F(u, \Delta)(a), F\left(u^{\text {rec }}, \Delta^{\text {res }}\right)$ for reconstructed $u$ and residual $\Delta(b)$; isoline levels: $F=0.2,0.5,1,2$, and 5
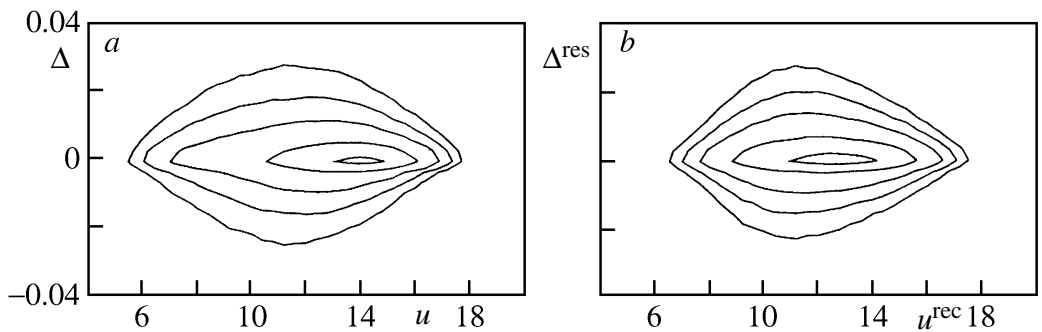

Fig. 11. Probability densities for the results of the numerical calculation: $F(u, \Delta)$ for the unfiltered data $(a), F\left(u^{\mathrm{rec}}, \Delta^{\mathrm{res}}\right)$ for reconstructed $u$ and residual $\Delta(b)$; isoline levels: $F=0.5,1,2,5$, and 10

For purposes of comparison, in Fig. 12 we have plotted the probability density of the residual field and the residual $u$ and reconstructed $\Delta$. Both figures are very similar to the corresponding figures for the placebo (for this reason the latter have not been reproduced) and indicate that the corresponding parameters are statistically independent.

The probability densities for the experimental data shown in Fig. 13 revealed multiple local maxima of $F(u, 0)$ which may indicate a small error (peak-locking) in these experimental data. In addition, as may be seen from Fig. 13a, the absolute maximum of $F(u, 0)$ is reached at a greater value of $u$ than the maximum of $F(u,-0.15)$, which confirms the presence of a statistical dependence between $u$ and $\Delta$. As distinct from the case of the numerical calculation data, filtration significantly weakens this dependence: in Fig. $13 b$ the maximum of $F(u, 0)$ is reached at a greater value of $u$ than the maximum of $F(u,-0.15)$. This also indicates the presence of a statistical dependence between the streaky structures and the true eddies, but in this case the difference in the location of the maxima may be within the error of the data measurements.

Summary. The existence of a relation between the streaky structures and other ordered structures observed in turbulent flows seems to be quite natural. However, this intuitive perception can lead to errors. Testing by means of a placebo makes it possible to ensure an objective interpretation data. The present investigation was restricted by two factors. Firstly, the extreme simplicity of the placebo used led to the separation of the streaky structures and the true eddies in the placebo by filtration turning out to be very rudimentary. Secondly, there was no possibility of changing the conditions of the numerical or physical experiment on the basis of the analysis carried out since only the data obtained earlier could be used. For this reason it remains unclear how far the imperfection of the filter used affected the results. In particular, it is unclear whether the statistical dependence observed between the streaky structures and the filtered eddies reflects the existence of a statistical dependence between these structures and the true eddies or whether this observation is entirely the result of incomplete filtration.

For the results of the numerical calculation and experiments it was found that, although filtration does not completely destroy the statistical dependence between the streaky structures and the eddies, it significantly weakens that dependence. Consequently, we may conclude that the dependence between the streaky structures and the true eddies of significance for the dynamics is weaker than the dependence between the streaky structures and the eddies directly visualized in the unfiltered field. 

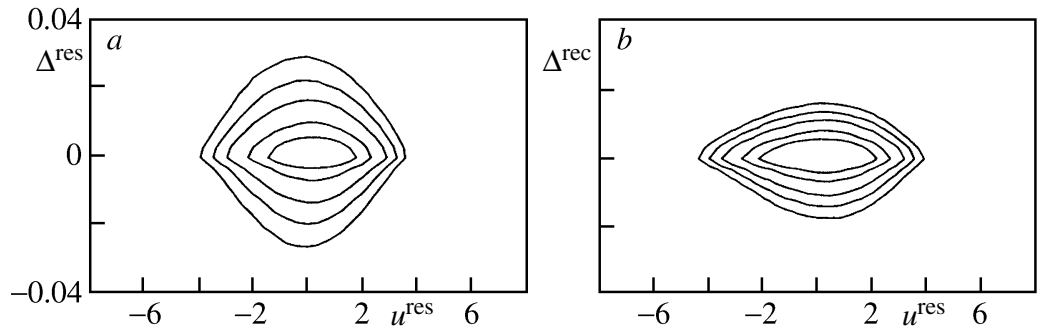

Fig. 12. Probability densities for the results of the numerical calculation: residual field $F\left(u^{\mathrm{res}}, \Delta^{\mathrm{res}}\right)(a), F\left(u^{\mathrm{res}}, \Delta^{\mathrm{rec}}\right)$ for residual $u$ and reconstructed $\Delta(b)$; isoline levels the same as in Fig. 11
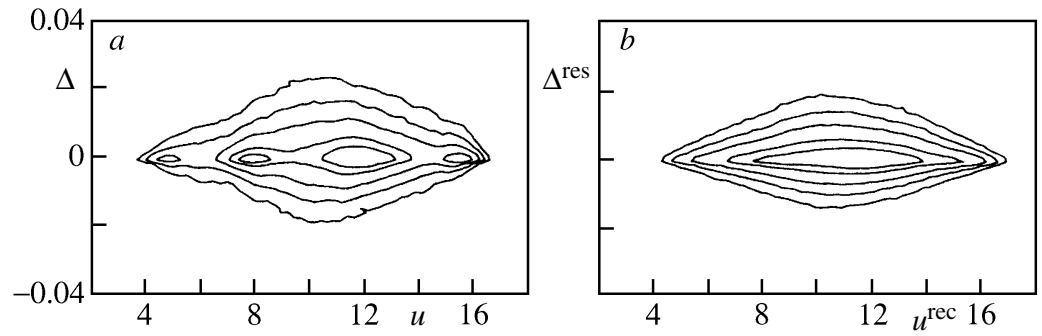

Fig. 13. Probability densities for the experimental data: unfiltered $F(u, \Delta)(a), F\left(u^{\text {rec }}, \Delta^{\text {res }}\right)$ for reconstructed $u$ and residual $\Delta(b)$; isoline levels the same as in Fig. 11

In both the cases considered it was found that the relation between the streaky structures and the eddies observable by means of Galilean decomposition and analysis of the swirling strength does not completely reflect the true interrelationship between the streaky structures and the other elements of the flow field. To a considerable degree, this relation is an artefact created by these eddy identification methods. We may conclude that the use of placebo techniques should be an obligatory element of any interpretation of the data on turbulent flow structure.

Part of this work was supported by Grant EPSRC GR/R64964/01 (UK Turbulence Consortium Grant).

\section{REFERENCES}

1. A. V. Boiko, G. R. Grek, A. V. Dovgal', and V. V. Kozlov, Development of Turbulence in Near-Wall Flows [in Russian], Nauka, Novosibirsk (1999).

2. S. I. Chernyshenko and M. F. Baig, "The mechanism of streak formation in near-wall turbulence," J. Fluid Mech., 44, 99-131 (2005).

3. R. J. Adrian, K. T. Christensen, and Z.-C. Liu, "Analysis and interpretation of instantaneous turbulent velocity fields," Experim. Fluids, 29, No. 3, 275-290 (2000).

4. M. S. Chong, A.E. Perry, and B. J. Cantwell, "A general classification of three-dimensional flow field," Phys. Fluids A, 2, No. 5, 765-777 (1990).

5. I. Marusic, "On the role of large-scale structures in wall turbulence," Phys. Fluids, 13, No. 5, 735-743 (2001).

6. A. Smirnov, S. Shi, and I. Celik, "Random flow generation technique for large eddy simulations and particledynamics modeling," Trans. ASME. J. Fluids. Engng., 123, No. 2, 359-371 (2001).

7. D. R. Osborne, J. C. Vassilicos, and J. D. Haigh, "One particle two-time diffusion in three-dimensional homogeneous isotropic turbulence," Phys. Fluids, 1y, No. 3, 03510.1-035104.11 (2005).

8. G. Kawahara, J. Jiménez, M. Uhlmann, and A. Pinelli, "The instability of streaks in near-wall turbulence," Аnnu. Res. Briefs, CTR, 155-170 (1998).

9. Z. Hu, Chr. J. Morfey, and N. D. Sandham, "Sound radiation in turbulent channel flows," J. Fluid Mech., 475, 269-302 (2003).

10. C. D. Tomkins and R. J. Adrian, "Spanwise structure and scale growth in turbulent boundary layers," J. Fluid Mech., 490, 37-74 (2003). 
11. P. R. Spalart, "Direct simulation of a turbulent boundary layer up to $\mathrm{Re}=1410$, , J. Fluid Mech., 187, 61-98 (1988).

12. S. Robinson, "Coherent motions in the turbulent boundary layer," Annu. Rev. Fluid Mech., 23, 601-639 (1991).

13. G. Berkooz, P. J. Holmes, and J. L. Limley, "The proper orthogonal decomposition in the analysis of turbulent flows," Аnпи. Rev. Fluid Mech., 25, 539-575 (1993).

14. Z. Hu and N. D. Sandham, "DNS databases for turbulent Couette and Poiseuille flow," Technical Report 01/04. 2002, AFM Research Group, SES, Univ. Southampton (2001).

15. M.-G. Di Cicca, G. Iuso, P. G. Spazzini, and M. Onorato, "Particle image velocimetry investigation of turbulent boundary layer manipulated by spanwise wall oscillations," J. Fluid Mech., 467, 41-56 (2002). 